\title{
Prehypertension or masked hypertension-which is responsible for target-organ damage?
}

Ying Chen, Yu-li Huang and Wei-yi Mai

We read the Review by Egan and StevensFabry (Prehypertension- prevalence, health risks, and management strategies. Nat. Rev. Cardiol. 12, 289-300; 2015) ${ }^{1}$ with great interest. We wish to discuss some views about screening for masked hypertension for risk stratification in prehypertension.

Epidemiological studies have shown that prehypertension is associated with increased risk of target-organ damage. ${ }^{1,2}$ However, data (described below) have shown that target-organ damage in prehypertension might be caused by masked hypertension, a condition in which individuals are found to have normal blood pressure (BP) in the clinic, but elevated out-of-office BP, measured by ambulatory $\mathrm{BP}$ monitoring (ABPM) or home BP monitoring (HBPM). ${ }^{3}$

First, masked hypertension is highly prevalent in individuals with prehypertension detected by BP measurement in the clinic. In the Masked Hypertension Study, ${ }^{4}$ ABPM revealed that $34.1 \%$ of participants determined by clinic BP measurement to have prehypertension, but only $3.9 \%$ of participants that had been classified as being normotensive ( $\mathrm{BP}<120 / 80 \mathrm{mmHg}$ ), had masked hypertension. The IDACO (International Database on Ambulatory blood pressure in relation to Cardiovascular Outcomes) study ${ }^{5}$ also showed that the prevalence of masked hypertension was higher among patients deemed by clinic BP measurement to be prehypertensive $(29.3 \%)$ than among those deemed to be normotensive (7.5\%). Similar results were observed by HBPM in the IDHOCO (International Database of HOme blood pressure in relation to Cardiovascular Outcome), ${ }^{6}$ which showed that prevalence of masked hypertension was $5.0 \%, 18.4 \%$, and $30.4 \%$ in patients identified by clinic BP measurement as having normotension, stage 1 (120-129/80-84 $\mathrm{mmHg}$ ) prehypertension, and stage 2 prehypertension (130-139/85-89 mmHg), respectively.

Second, masked hypertension is associated with subclinical cardiovascular disease in prehypertension. Manios et al. showed that prehypertensive patients with masked hypertension had greater carotid intimamedia thickness than either prehypertensive patients without masked hypertension or normotensive participants. ${ }^{7}$ One study found that masked hypertension might also be associated with endothelial dysfunction in prehypertension. ${ }^{8}$ However, in another study, although left ventricular mass index in participants with prehypertension or masked hypertension was greater than that in normotensive participants, no significant difference was observed among participants with prehypertension without or with masked hypertension. ${ }^{9}$

Third, masked hypertension is the important cause of cardiovascular disease in individuals with prehypertension. Pierdomenico et al. first reported that among individuals with prehypertension, cardiovascular risk was higher in those with masked hypertension than in those without masked hypertension. ${ }^{10}$ The IDACO study ${ }^{5}$ showed that, after adjustment for multiple confounding factors (including clinic BP), individuals identified by clinic $\mathrm{BP}$ measurement to have normotension or prehypertension, but by ABPM to have masked hypertension, were three times as likely to experience stroke as individuals who had true normotension (deemed to be normal by both clinic BP measurement and by $A B P M)$; stroke risk did not significantly differ between the two groups with masked hypertension. Similarly, in a participantlevel meta-analysis of risk stratification by HBPM, compared with true normotension (BP deemed normal by both clinic BP measurement and HBPM), all-cause mortality and cardiovascular end points were increased in all participants with masked hypertension who had been identified as having normotension, stage 1 prehypertension, or stage 2 prehypertension by $\mathrm{BP}$ measurement in the clinic. ${ }^{6}$ Again, the risks in the three groups of participants with masked hypertension were not significantly different. ${ }^{\circ}$

In conclusion, although studies have shown that prehypertension is associated with increased risk of target-organ damage, the actual concern about this health risk is not the mild elevation in BP measured in the clinic, but the underestimation of masked hypertension. Out-of-office BP measurements should be recommended for risk restratification in individuals identified in the clinic as being prehypertensive.

The Second Out-Patient Department (Y.C.) and the Department of Cardiology (Y.-L.H.), the First People's Hospital of Shunde, Penglai Road 1, Daliang Town, Shunde District, Foshan 528300, China. Department of Cardiology, the First Affiliated Hospital of Sun Yat-sen University, 58 Zhongshan Road 2, Guangzhou 510080, China (W.-Y.M.). Correspondence to: W.-Y.M. wymai@hotmail.com

\section{Competing interests}

The authors declare no competing interests.

1. Egan, B. M. \& Stevens-Fabry, S. Prehypertension - prevalence, health risks, and management strategies. Nat. Rev. Cardiol. 12, 289-300 (2015).

2. Huang, Y. et al. Prehypertension and the risk of stroke: a meta-analysis. Neurology $\mathbf{8 2}$, 1153-1161 (2014).

3. Pickering, T. G., Davidson, K., Gerin, W. \& Schwartz, J. E. Masked hypertension. Hypertension 40, 795-796 (2002).

4. Shimbo, D., Newman, J. D. \& Schwartz, J. E. Masked hypertension and prehypertension: diagnostic overlap and interrelationships with left ventricular mass: the Masked Hypertension Study. Am. J. Hypertens. 25, 664-671 (2012).

5. Brguljan-Hitij, J. et al. Risk stratification by ambulatory blood pressure monitoring across JNC classes of conventional blood pressure. Am. J. Hypertens. 27, 956-965 (2014).

6. Asayama, K. et al. Risk stratification by selfmeasured home blood pressure across categories of conventional blood pressure: a participant-level meta-analysis. PLoS Med. 11, e1001591 (2014).

7. Manios, E. et al. Impact of prehypertension on carotid artery intima-media thickening: actual or masked? Atherosclerosis 214, 215-219 (2011).

8. Veerabhadrappa, P. et al. Endothelial-dependent flow-mediated dilation in African Americans with masked-hypertension. Am. J. Hypertens. 24, 1102-1107 (2011).

9. Sobrino, J., Domenech, M., Camafort, M., Vinyoles, E. \& Coca, A. Prevalence of masked hypertension and associated factors in normotensive healthcare workers. Blood Press. Monit. 18, 326-331 (2013).

10. Pierdomenico, S. D. et al. Prognostic relevance of masked hypertension in subjects with prehypertension. Am. J. Hypertens. 21, 879-883 (2008). 